\title{
COLOR MIXING SIMULATOR FOR DISPLAY SURFACES BASED ON HUMAN COLOR VISION
}

\author{
Takako Nonaka \\ Ryukoku University \\ Otsu, Shiga, 520-2194, Japan \\ nonaka@rcics.hrc.ryukoku.ac.jp
}

\author{
Morimasa Matsuda \\ Mitsubishi Electric Microcomputer \\ Application Software Co., Ltd. \\ Nagaokakyo, Kyoto, 617-8550, Japan \\ morimatsu@river.ocn.ne.jp
}

\author{
Tomohiro Hase \\ Ryukoku University \\ Otsu, Shiga, 520-2194, Japan \\ hase@rins.ryukoku.ac.jp
}

\section{KEYWORDS}

Color mixing process, pixel structures, viewing distance, perceived color.

\begin{abstract}
This paper proposes a simulator that analyzes the color mixing process in large display units based on human color vision.

The authors have previously proposed calculation model for the color mixing process. With this model, we can calculate the viewing distance at which humans distinguish the pixel structure, but the model does not give us the appearance of any particular image.

Therefore, the proposed simulator enables us to understand how mixed colors are perceived without actually manufacturing a large display unit. It also supplies the output of the resulting image.

First, the simulator makes test samples that reproduce the pixel structure. Next, the spatial frequency characteristics of each of the R, G and B pixel dots on the display surface are calculated. Thirdly, the visual characteristics in the spatial-frequency domain for each color are realized as a transfer function of a low pass filter, where the viewing distance is used as a parameter. Fourthly, in the spatial-frequency domain, the display surface characteristics are multiplied with the characteristics of human vision for each color. Finally, the simulator converts the spatial-frequency characteristics to the spatial characteristics, and composes the appearance of each color to show the resulting color mixing image.

Using this simulator, we tried to produce the appearance of images at the different viewing distances. As a result, we found that the longer the viewing distance becomes, the less recognizable are the pixel dots and they look to have a more uniform, mixed color. These results agree qualitatively with the appearance of images on the screens of actual large display units and prove the validity of our simulator.
\end{abstract}

\section{INTRODUCTION}

A large display unit clearly shows the rows and lines of pixels and their structure when viewed from nearby, which prevents the displayed images from appearing with their colors evenly mixed. In order to discuss this inconvenience in visual perception, the authors have previously reported a calculation model that considers the characteristics of the display surface and human vision. This model treats the color mixing in images shown on display units as a phenomenon in which repeated patterns of pixel arrays are influenced by the visual characteristics in proportion to the viewing distance from the display surface. With this model, we can calculate the viewing distance at which humans do not recognize the pixel structure. However the model does not give us the appearance of any particular image and does not support easy understanding of the color mixing results.

Therefore, this paper proposes a simulator that can output how the image appears based on this calculation model.

First, a color mixture model based on the visual characteristics and how to realize the model in simulation programs are discussed in the second section. Next, to verify the validity of the proposed simulator, some simulation results are shown and discussed.

\section{COLOR MIXTURE MODEL}

\section{A calculation model of color mixing}

The authors have previously proposed a modulation transfer function (MTF) model of the assimilation phenomenon based on visual characteristics (Nonaka et al. 2005). This is a model based on the spatial frequency response concerning luminance, and corresponds to Area II shown in Figure 1 (Hase et al. 1993).

In this area, patterns are identified which react to the contrast of luminance in the presented samples. When discussing the assimilation phenomena of brightness and interference impressions caused by the pixel structures of display units, this area is one of contention. On the other hand, the color mixing phenomenon is based on the human characteristics of color vision. The phenomenon is influenced by the spatial frequency response concerning chrominance signals, and corresponds to Area I in Figure 1.

This section describes the proposal of a color mixing simulator on the basis of these relationships.

First, an outlines of the calculation model and the simulator are discribed. Next, the spatial-frequency characteristics of human color vision are expressed. Thirdly, the color mixing process of R-G stripes is interpreted by using the color mixing model. 


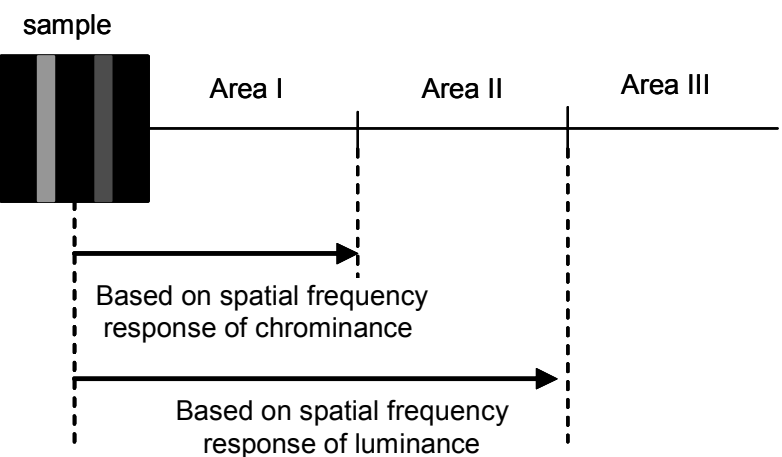

Figure 1. Relationship between appearance of color images and human visual characteristics

Figure 2 shows the proposed calculation model of a color mixture on the basis of these relationships (Nonaka et al. 2006). This model is based on human color vision.

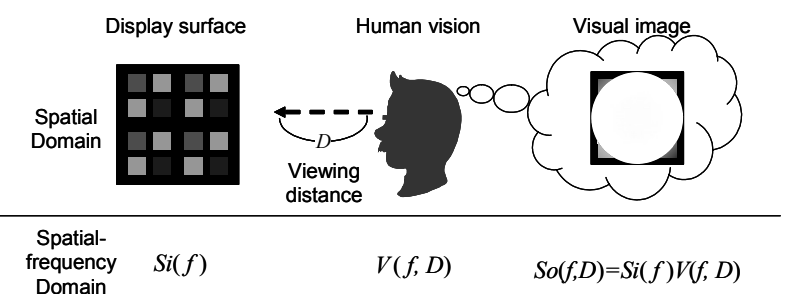

Figure 2. Proposed model of the color mixture based on human color vision.

The proposed model treats the juxtaposed color mixture as follows; the color signals of the sample image attenuate because of color vision, and the attenuated signals overlap each other. In this paper, the characteristics of the color signals are named $\mathrm{Si}$, and the characteristics of human color vision are named $V$. This model treats the color mixing in images shown on display units as a phenomenon in which repeated patterns of pixel arrays are influenced by the visual characteristics in proportion to the viewing distance from the display surface.

With this model, we can calculate the viewing distance at which humans do not recognize the pixel structure. However, the model does not give us the appearance of any particular image.

With this in mind, this study has created a simulator that can supply an output of how the image appears based on this calculation model.

\section{Analytical simulator of color mixing process}

Figure 3 shows the relationship between the calculation model and the processing flows of the proposed simulator.

Figure 4 shows the software configuration of the proposed simulator.

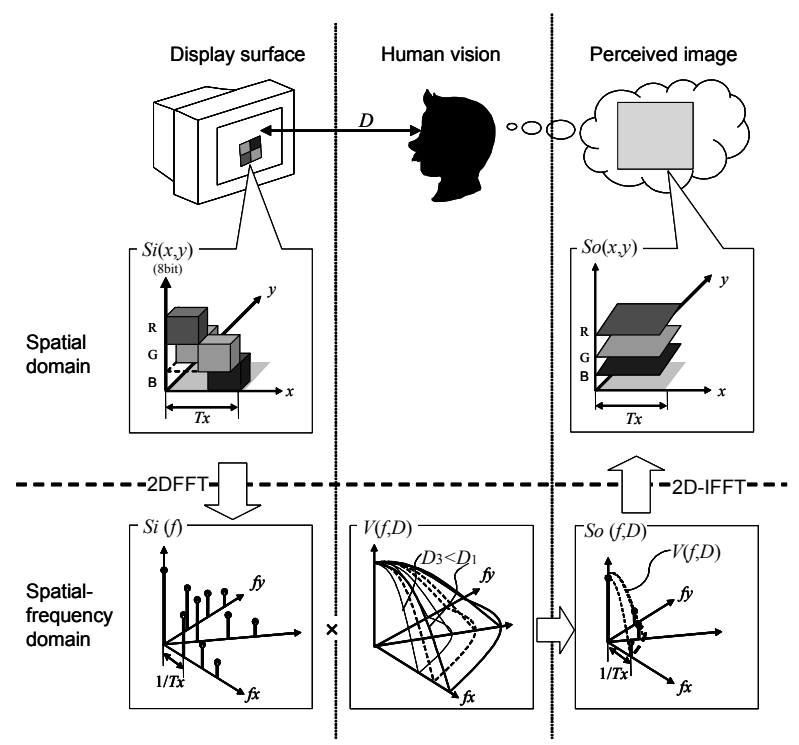

Figure 3. Processing flows of the proposed simulator based on the calculation model for color mixing

First, the simulator makes test samples that reproduce the pixel structure. Next, the spatial frequency characteristics of each of the R, G and B pixel dots on the display surface are calculated by two-dimensional fast Fourier transform (2D-FFT). Thirdly, the visual characteristics in the spatial-frequency domain for each color of $\mathrm{R}, \mathrm{G}$ and $\mathrm{B}$ are realized as a transfer function of a low pass filter, where the viewing distance is used as a parameter. Fourthly, in the spatial-frequency domain, the display surface characteristics are multiplied with characteristics of human vision for each color of $R, G$ and $B$. Finally, the simulator converts the spatialfrequency characteristics to the spatial characteristics by two-dimensional inverse Fourier transform (2D-IFFT), and composes the appearance of each color to show the resulting color mixing image.

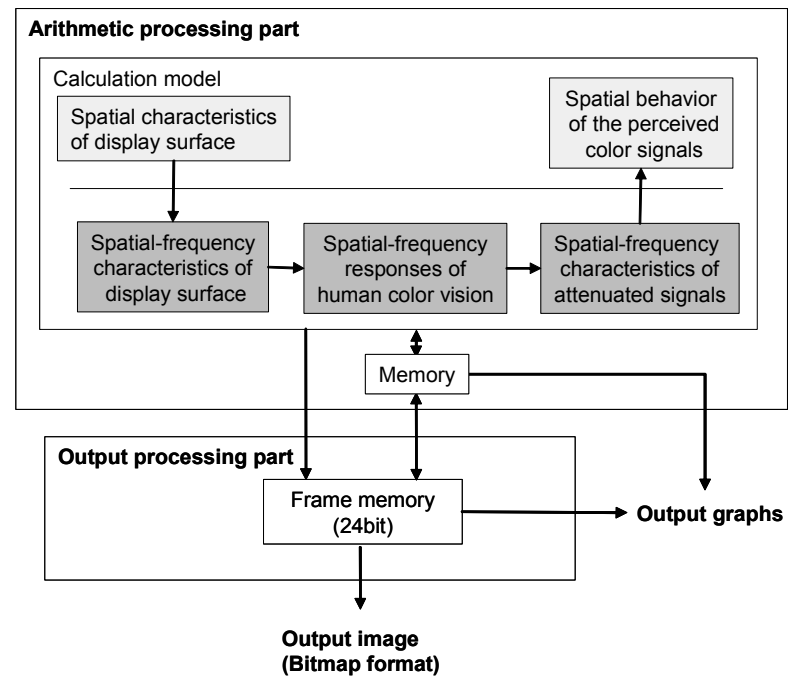

Figure 4. Software configration of the proposed simulator 


\section{Modeling human color vision}

The characteristics of human color vision are considered here as spatial frequency responses to color. There have been experimental reports that the shape of the contrast sensitivity function of human color vision is a band pass filter (Cavonius and Estevez, 1975).

However, the focus of this discussion is the attenuating behavior of power spectrum densities of the display surfaces influenced by the high frequency characteristics of human color vision.

Therefore, the spatial frequency responses of color vision are expressed in this paper as $V(v)$ with the following Butterworth LPF.

$$
V(v)=\frac{1}{\sqrt{1+\left(\frac{v}{v_{C}}\right)^{2 k}}}
$$

Here, $v$ [cycle/degree] is the frequency of the color pattern on the display surface according to the viewing angle. The order parameter $k$ controls an attenuation slope of the LPF, and the reasonable choice for human vision is 1 (Hase et al. 1993).

Also, $v_{\mathrm{C}}$ [cycle/degree] means the cutoff frequency of each color when the amplitude becomes half of the original. In this paper, the $v_{\mathrm{R}}, v_{\mathrm{G}}$, and $v_{\mathrm{B}}$, of red, green, and blue, i.e. the primary colors of light, are given the following values with reference to Kurahashi's report (Kurahashi 1986).

$$
\left.\begin{array}{l}
v_{R}=5.0 \\
v_{G}=8.8 \\
v_{B}=2.4
\end{array}\right\}
$$

The original spatial frequency $v$ [cycles/degree] of color vision is converted by using the frequency of the display surface $f$ [cycle $/ \mathrm{m}]$ and the viewing distance $D$ [m] into as follows (Hase et al. 1993):

$$
v=\frac{1}{2 \tan ^{-1}\left(\frac{1}{2 f D}\right)}
$$

Where, the viewing distance $D[\mathrm{~m}]$ means the distance between the sample image and the observer. It is used as a parameter in this formula.

Therefore, Formula 1 can be expressed as follows:

$$
V(v)=\frac{1}{\sqrt{1+\left\{2 v_{C} \tan ^{-1}\left(\frac{1}{2 f D}\right)\right\}^{-2}}}
$$

Figure 5 shows simulation examples of the output graph of $V(f, D)$ for $\mathrm{R}, \mathrm{G}$, and $\mathrm{B}$ when the viewing distance $D$ is $5 \mathrm{~m}$.

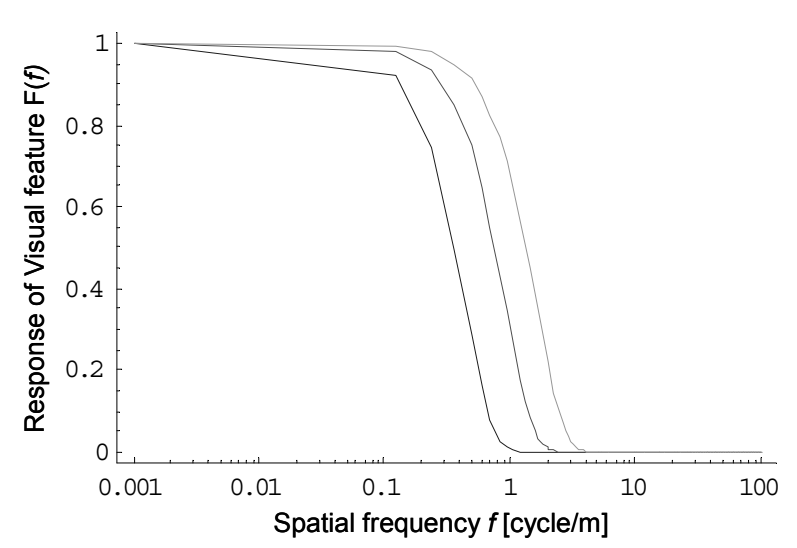

Figure 5. Examples of simulation results of human color vision $V(f, D=5)$ in the spatial-frequency domain

Figure 6 shows the output graph of the proposed simulator for green only, using the viewing distances $D$ of $1,2,5$ and $10 \mathrm{~m}$. When the viewing distance is longer, the characteristics of visual LPF work better.

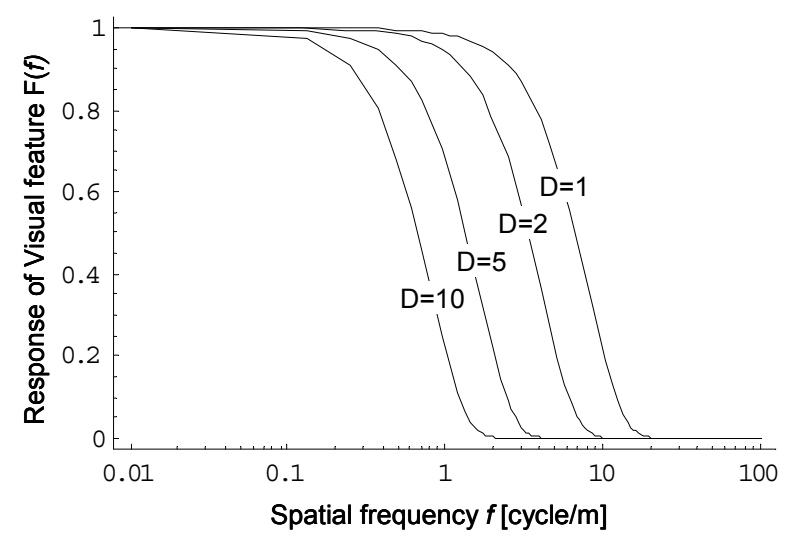

Figure 6. Examples of simulation results of human color vision with the viewing distance $D$ as parameters (the color is green).

\section{Interpretation of color mixing phenomenon by the proposed model}

The spread of color signals in the spatial domain attenuated by the influence of the LPF of color vision was calculated using this proposal. Therefore, the area where the attenuated signals overlap each other is interpreted as mixed color.

The behavior of the signal is obtained as a multiplication of the Fourier coefficients $S i(f)$ of the signal on the sample surface and the visual characteristics $V(f)$ in the spatial-frequency domain. The shape of the waves of the perceived color signals in the spatial domain are calculated by 2D-IFFT of $S i(f) \times$ $V(f, D)$, So $(f, D)$. This signal seems to indicate the juxtaposed mixture of colors. The simulator realizes the calculation by using 2D-IFFT for image processing. Figure 7 shows how the signal behaves under the influence of human visual characteristics. The 
appearance when the red signal mixes with the green signal and looks yellow is also shown in Figure 7.

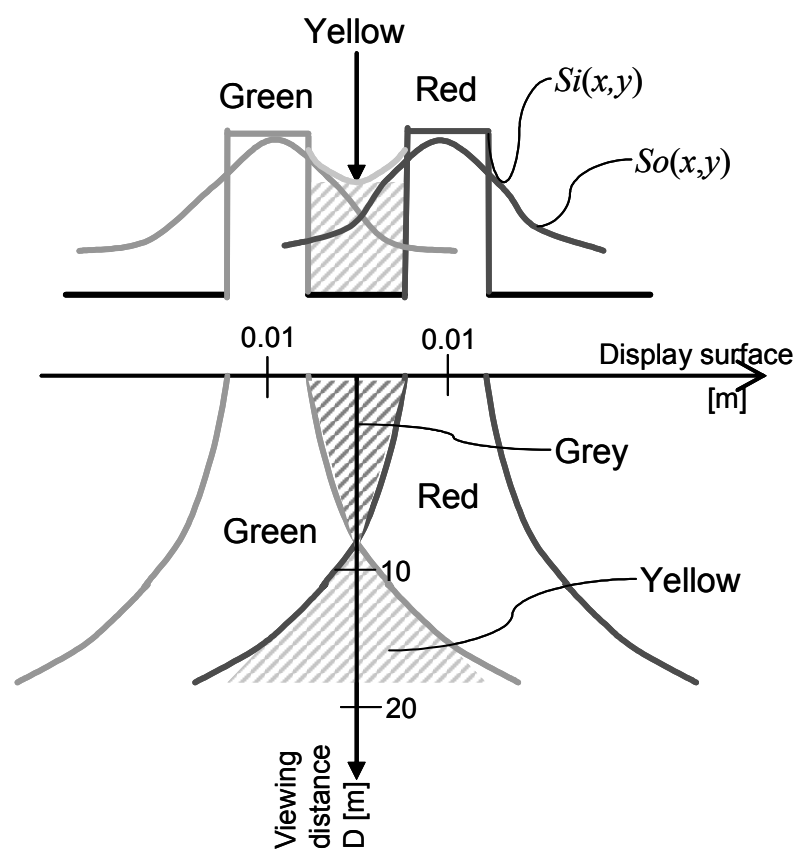

Figures 7. The behavior of color signals in human color vision in the spatial domain.

When the viewing distance is longer, the waveform of the color signal is broader in the spatial domain. It was assumed that both the original signals of red and green overlapped with each other.

These results show that the apparent yellow area increases according to the viewing distance.

\section{VERIFICATION OF PROPOSAL}

In order to verify the proposed model, some simulation results are discussed here. Using this simulator, we tried to produce the appearance of images at the viewing distances $D$ of $5 \mathrm{~m}$ and $25 \mathrm{~m}$ for test samples of $\mathrm{R}$ and B stripes with the width of the color pixel dots being 10 pixels of $2.8 \mathrm{~mm}$ and the dot periods being 20 pixels of $5.6 \mathrm{~mm}$. Figure 8 shows the resulting images.

For the distance of $5 \mathrm{~m}$, the produced image was identified as having both $\mathrm{R}$ and $\mathrm{B}$ colors viewed in their primary colors. At the distance of $25 \mathrm{~m}$, the striped pattern became less recognizable, and the entire image looked to be magenta.

This demonstrates that the longer the viewing distance becomes, the less recognizable are the patterns of $\mathrm{R}, \mathrm{G}$ and $\mathrm{B}$ color by the pixel array and they look to have a more uniform, mixed color. The results of the simulation agree qualitatively with the appearance of images on the screens of actual large display units. We believe that the above results prove the validity of our suggested simulator.

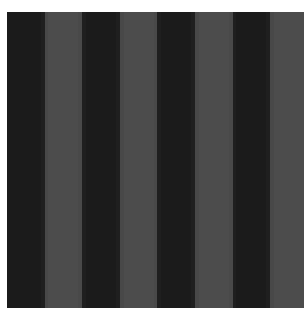

(a) Original test image

(b) Output image of simulation

\section{CONCLUSIONS}

This paper has proposed an analytical simulator for the color mixing process by considering human color vision. The simulator is based on a proposed calculation model that treats a color mixture as color signals of a sample image which attenuate and overlap with each other in human color vision.

First, the characteristics of the display surfaces were calculated in this simulator by 2D-FFT, $S i(f)$. Secondly, the characteristics of human color vision, $V(f, D)$, were approximated by the Butterworth LPF. Thirdly, a relational model of human vision and the appearance of the display surface, So $(f, D)$ was expressed as multiplication of $\operatorname{Si}(f)$ and $V(f, D)$. Finally, the resulting appearances of the color mixture were calculated by 2D-IFFT.

As an example, the appearance when $R$ mixes with $G$ and looks yellow was interpreted as being the longer the viewing distance becomes, the broader the waveforms of $\mathrm{R}$ and $\mathrm{G}$ are in the spatial domain. In other words, the apparent yellow area increases according to the viewing distance.

Additionally, we tried to produce the appearance of color mixtures at various viewing distances by using this simulator. The results showed that the longer the viewing distance becomes, the less recognizable are the pixel dots of R, G and B and they look to have a more uniform, mixed color.

These results agree qualitatively with the appearance of images on the screens of actual large display units and prove the validity of our suggested simulator.

\section{REFERENCES}

Cavonius C. R. and O. Estevez. 1975. "Contrast Sensitivity of Indivisual Colour Mechanisms of Human Vision", Journal of Physiology, 248, 649-662.

Kurahashi K. 1986. "Visual Color Shifts in Spatial Array of Three Primary Colors", Journal of the Institute of Television Engineers of Japan, Vol. 40, No. 5, 392-397 (in Japanese).

Hase T., N. Shiramatsu, and S. Tomita. 1993. "Effects on the Mosaic Pixel Structure on Display Images", Journal of the Institute of Television Engineers of Japan, Vol. 47, No. 3, 356-363 (in Japanese).

Nonaka, T., M. Matsuda and T. Hase. 2005. "Modulation Transfer Function Model of Assimilation Phenomenon Based on Visual Characteristics", Proceeding Book of AIC 05 (May), 983-986. 
Nonaka T., M. Matsuda and T. Hase. 2006. "Additive Color Mixing Model Based on Human Color Vision for Bayertype Pixel Structures", Proceeding book of ISCE 2006 (June), 98-99.

\section{AUTHOR BIOGRAPHIES}

Takako Nonaka was born in 1975 in Osaka, Japan. She received her BS, MS and Ph.D. degrees from the Kyoto Institute of Technology, Japan in 1998, 2000 and 2004. She engaged in research on modeling the sensory evaluation for 2D design as a visiting researcher of Tomas Bata University in Zlin, the Czech Republic from April to September 2004. She was a postdoctoral research fellow of High Technology Research Center at Ryukoku University from October 2004 to March 2006. She has been a postdoctoral research fellow of the Research Center for Information Communication Systems at Ryukoku University since May 2006.
Morimasa Matsuda was born in 1964 in Hyogo, Japan. He received a B.S. degree from Kinki University, Japan, in Electronics Engineering in 1987. In 1987 he joined the Television Technology Division of the Kyoto Works of Mitsubishi Electric Microcomputer Application Software Co. Ltd.

Tomohiro Hase was born in 1953 in Gifu, Japan. He received a B.S. degree from Fukui University, and M.S. and Ph.D. degrees from Shizuoka University, Japan. All degrees were in Electronics Engineering, and were received in 1977, 1979 and 1995 respectively. In 1979, he joined Mitsubishi Electric Corporation, and engaged in research into signal processing for audio visual equipment and microprocessor applications. He has been a professor of the Faculty of Science and Technology of Ryukoku University since 2000 